P206 (continued)

financial pressures, difficult choices made when stretching limited income, difficulties in improving one's situation and emotional stresses and frustrations when living on a limited budget. The majority of interns (88\%) successfully met the TMPSC and 75\% utilized alternative forms of food access (ex. food pantries, soup kitchens, gardening).

Conclusions and Implications: The TMPSC provides interns with a social simulation experience to prepare and sensitize them for working in Maine, the state that has the third highest rate of very low food security in the United States.

Funding: None

\section{P207 Undergraduate Nutrition Students Gain Knowledge and Skills by Leading Grocery Tours}

Adrienne Fraczkowski, University of Delaware;

Maria Tsugranes; Chengshun Richard Fang, PhD, RD;

Cassandra Umile, $B S, R D$, Kenny Family ShopRites;

Sandra Baker, EdD, LDN, RD, bakers@udel.edu, University

of Delaware, 032 Carpenter Sports Building, Newark, DE 19716

Objective: Evaluate the effectiveness of a grocery store tour program with undergraduates as tour leaders.

Theory, Prior Research, Rationale: Adult Learning Theory maintains adults learn through practical, problem oriented approaches. Grocery tours provide hands-on education but little has been published about the possible benefits for undergraduates leading tours.

Description: Target audience were upperclassmen, nutrition majors and community participants in grocery tours University faculty and a supermarket dietitian collaborated to provide this experience for nutrition student volunteers $(n=20)$. Using the Produce for Better Health's Store Training Guide, the retail RD led a 5-hour training. Tours were advertised through the store. In pairs, students conducted nine tours averaging 2-3 participants.

Evaluation: Almost all $(n=17)$ of the students completed an IRB approved survey at completion of the project. Most $(n=13)$ had little to no experience providing nutritional education prior to this experience. Over half indicated conducting the tours increased their confidence in providing nutrition information and most $(n=12)$ felt more confident in public speaking. Most gained knowledge about the health benefits of fruits and vegetables. Almost all $(n=16)$ gained understanding about the retail dietitian career. Most indicated more effort should be spent advertising tours in the future. Community participants $(n=25)$ also completed an IRB approved post-experience survey and responses were very favorable. In write-in responses, participants indicated they enjoyed the informal opportunity to ask questions.

Conclusions and Implications: Students benefited from this hands on experience. The nutrition program plans to continue the partnership which gives students learning opportunities and provides nutrition education for the public.

Funding: Produce for Better Health

\section{P208 Where Did They Go Wrong? Identifying Student Strategies Used in Analytic Thinking, Evaluating Data, and Problem Solving}

Alyce Fly, PhD, afly@indiana.edu, Indiana University, 1025 East 7th Street PH 116, Bloomington, IN 47405; Krisha Thiagarajah, PhD, Indiana University; Lisa Kurz, PhD

Objective: This formative study was undertaken to identify and evaluate strategies students use solving nutrition problems so that ineffective strategies can be addressed in instruction to improve future course outcomes.

Theory, Prior Research, Rationale: Exploring strategies students use in answering nutrition questions will help instructors tailor instruction to improve learning.

Description: One hundred and twenty-three student volunteers (129 undergraduates enrolled) from 2 sections of an introductory nutrition course completed a 10-item quiz administered at the end of the semester. Students included nutrition majors and nonmajors seeking general education science credits. At this university general education science courses must demonstrate analytical thinking, problem solving, and interpreting/evaluating data. These types of learning are incorporated into topics on healthy eating, introductions to macronutrients, micronutrients, and food safety, with face-to-face instruction for 16 weeks.

Evaluation: Students described methods used to solve 3 quiz items. Investigators reviewed methods and the correctness of the solution. To interpret body mass index values, students who used a table (48\%) were correct more often than those relying on memory (51\%). To identify food group deficiencies given relevant data, most students partitioned food to groups (71\%) but only one-fifth obtained necessary information from charts defining quantities and specific recommendations (21\%). To calculate Estimated Energy Requirement, students used the given formula $(70 \%)$ or guessed $(20 \%)$. Over $60 \%$ of students using the formula gave incorrect answers due to difficulty with computations or converting units.

Conclusions and Implications: Encouraging students to use appropriate tables should increase success over relying on memory. Mastering unit conversions and reviewing order of operations should improve problem solving successes.

Funding: Indiana University

\section{P209 Exploring Relationships between Group Readiness and Implementation of Nutrition and Physical Activity Policy among Community Sectors}

Deana A. Hildebrand, PhD, RD, SNS, deana.hildebrand@ okstate.edu, Oklahoma State University, Human Sciences, Stillwater, OK 74078; Jeremy Humphrey, MS, Oklahoma State University; Healther M. Ross, MS, University of Oklahoma; Rebekah R. Rhoades, MS 